\title{
The integral mean of the sum-of-digits function of the Ostrowski expansion
}

\author{
by \\ Luís RoÇADAs (Vila Real)
}

1. Introduction and statement of results. Let $G=\left(G_{j}\right)_{j \geq 0}$ be a strictly increasing sequence of integers with $G_{0}=1$. Then every nonnegative integer $N$ has a unique $G$-ary expansion $N=\sum_{j \geq 0} b_{j}(N) G_{j}$ with integer digits $b_{j}(N)$ provided that $\sum_{j<k} b_{j}(N) G_{j}<G_{k}$. The sum-of-digits function $t_{G}(N)$ is given by

$$
t_{G}(N)=\sum_{j \geq 0} b_{j}(N)
$$

There are several papers concerning the distribution of $t_{G}(N)$ for fixed $G$, e.g. [1] and [2]. In [3] this function is studied by fixing $N$ and considering the average values of $H^{-1} \sum_{g=2}^{H} t_{G}(N)$ with $G=\left(g^{j}\right)_{j \geq 0}$.

Let $\Omega$ be the set of all irrational numbers in the interval $[0,1]$. Then every $\alpha \in \Omega$ has a unique continued fraction expansion $\alpha=\left[0, a_{1}(\alpha), a_{2}(\alpha), \ldots\right]$ with convergents $p_{n}(\alpha) / q_{n}(\alpha)$. Given $N$, using the sequence $G=\left(q_{j}(\alpha)\right)_{j \geq 0}$, we can obtain uniquely determined integers $m(N, \alpha)$ and $b_{i}(N, \alpha)$ (if it is clear from the context we omit the dependence on $\alpha$ in $q_{i}, m$ and $b_{i}$ and the dependence on $N$ in $m$ and $\left.b_{i}\right), 0 \leq i \leq m$, with the following properties:

(1) $N=\sum_{i=0}^{m} b_{i} q_{i}$.

(2) $b_{m}>0$ and $0 \leq b_{i} \leq a_{i+1}$ for $0 \leq i \leq m$.

(3) If $0<i \leq m$ and $b_{i}=a_{i+1}$ then $b_{i-1}=0$. Furthermore $b_{0}<a_{1}$.

The expansion $N=\sum_{i=0}^{m} b_{i} q_{i}$ is called the Ostrowski expansion of $N$ to base $\alpha$. In analogy with the classical situation in [3] we study, for fixed $N$, the sum-of-digits function $s_{N}(\alpha)$ defined by $s_{N}(\alpha)=\sum_{i=0}^{m} b_{i}$ and the corresponding mean $\int_{0}^{1} s_{N}(\alpha) d \alpha$. More precisely, our main goal is to prove, using some techniques and ideas from [5], the following theorem.

2000 Mathematics Subject Classification: Primary 11K16, 11K50, 11K55, 11A55.

Key words and phrases: Ostrowski expansion, sum-of-digits function, metrical results. 
Theorem. For $N \in \mathbb{N}$,

$$
\int_{0}^{1} s_{N}(\alpha) d \alpha=\frac{3}{\pi^{2}} \log ^{2} N+O\left((\log N)^{3 / 2} \log \log N\right) .
$$

For the proof of the Theorem we proceed as follows: We start by proving, for $b_{i}>0$, the formulas $b_{i}=a_{i+1}\left\{N q_{i} \alpha\right\}+O(1)$ for $2 \mid i$, and $b_{i}=$ $a_{i+1}\left(1-\left\{N q_{i} \alpha\right\}\right)+O(1)$ for $2 \nmid i$. As $b_{i}=0$ only for $\alpha$ in a small set $A_{N, i}$, by integrating these formulas we get the relation

$$
\int_{0}^{1} b_{i}(\alpha) d \alpha=\frac{1}{2} \int_{0}^{1}\left(1+\frac{q_{i-1}(\alpha)}{q_{i}(\alpha)}\right) \log ^{+}\left(\frac{N}{q_{i}(\alpha)}\right) d \alpha+O(1) .
$$

Note that in contrast to $\int_{0}^{1} a_{i+1}(\alpha) d \alpha=\infty$ the corresponding integral over $b_{i}(\alpha)$ is finite. Finally we calculate $\int_{0}^{1} s_{N}(\alpha) d \alpha$ using the asymptotic relation

$$
\begin{aligned}
\sum_{i \geq 0} \int_{0}^{1}\left(1+\frac{q_{i-1}(\alpha)}{q_{i}(\alpha)}\right) \log ^{+}\left(\frac{N}{q_{i}(\alpha)}\right) d \alpha & \\
& =\frac{6}{\pi^{2}} \log ^{2} N+O\left((\log N)^{3 / 2} \log \log N\right) .
\end{aligned}
$$

2. Definitions and notations. For $\mathbf{a}=\left(a_{n}\right)_{n \in \mathbb{N}} \in \mathbb{N}^{\mathbb{N}}$ define sequences $\left(P_{n}(\mathbf{a})\right)_{n \in \mathbb{Z}_{+}}$and $\left(Q_{n}(\mathbf{a})\right)_{n \in \mathbb{Z}_{+}}$by $P_{0}(\mathbf{a})=0, Q_{0}(\mathbf{a})=1, P_{1}(\mathbf{a})=1$, $Q_{1}(\mathbf{a})=a_{1}$, and

$$
P_{k+1}(\mathbf{a})=a_{k+1} P_{k}(\mathbf{a})+P_{k-1}(\mathbf{a}), \quad Q_{k+1}(\mathbf{a})=a_{k+1} Q_{k}(\mathbf{a})+Q_{k-1}(\mathbf{a}), \quad k \geq 1 .
$$

Then $P_{k}(\mathbf{a})$ and $Q_{k}(\mathbf{a})$ depend at most on $a_{1}, \ldots, a_{k}$; hence we may write $P_{k}\left(a_{1}, \ldots, a_{k}\right)$ for $P_{k}(\mathbf{a})$ and $Q_{k}\left(a_{1}, \ldots, a_{k}\right)$ for $Q_{k}(\mathbf{a})$. If $\alpha=\left[0 ; a_{1}, \ldots\right]$, then for $k \geq 0, p_{k}(\alpha)=P_{k}\left(a_{1}, \ldots, a_{k}\right)$ and $q_{k}(\alpha)=Q_{k}\left(a_{1}, \ldots, a_{k}\right)$.

For $k \in \mathbb{Z}_{+}$and $\mathbf{a} \in \mathbb{N}^{k+1}$ let $J(\mathbf{a}):=\left\{\alpha \in \Omega: 1 \leq j \leq k+1 \Rightarrow a_{j}(\alpha)=a_{j}\right\}$. Then

$$
J(\mathbf{a})= \begin{cases}\left(\frac{P_{k+1}(\mathbf{a})+P_{k}(\mathbf{a})}{Q_{k+1}(\mathbf{a})+Q_{k}(\mathbf{a})}, \frac{P_{k+1}(\mathbf{a})}{Q_{k+1}(\mathbf{a})}\right) & \text { if } k \text { is even, } \\ \left(\frac{P_{k+1}(\mathbf{a})}{Q_{k+1}(\mathbf{a})}, \frac{P_{k+1}(\mathbf{a})+P_{k}(\mathbf{a})}{Q_{k+1}(\mathbf{a})+Q_{k}(\mathbf{a})}\right) & \text { if } k \text { is odd },\end{cases}
$$

but in any case, if $\lambda$ denotes the Lebesgue measure on $\Omega$,

$$
\lambda(J(\mathbf{a}))=\frac{1}{Q_{k+1}(\mathbf{a})\left(Q_{k+1}(\mathbf{a})+Q_{k}(\mathbf{a})\right)} .
$$

For $x>0$ we define $\log ^{+} x=\max \{\log x, 0\}$. For a real number $x$ let $\{x\}:=x-[x]$ be the fractional part of $x$. 
3. Auxiliary results. We start by proving, for $b_{i}>0$, the formulas

$$
b_{i}= \begin{cases}a_{i+1}\left\{N q_{i} \alpha\right\}+O(1) & \text { if } 2 \mid i, \\ a_{i+1}\left(1-\left\{N q_{i} \alpha\right\}\right)+O(1) & \text { if } 2 \nmid i .\end{cases}
$$

These formulas are valid for $\alpha \in \Omega$ except for $\alpha \in A_{N, i}=\left\{\alpha \in \Omega: A_{i}<0\right\}$ if $i$ is even, and except for $\alpha \in A_{N, i}=\left\{\alpha \in \Omega: A_{i}>0\right\}$ if $i$ is odd. Then we calculate the integral of $a_{i+1}\left\{N q_{i} \alpha\right\}$ and $a_{i+1}\left(1-\left\{N q_{i} \alpha\right\}\right)$ over $[0,1]$. The rest of the section is devoted to obtaining an upper bound of the integral of $a_{i+1}(\alpha)$ over $A_{N, i}$.

For $i, j \in \mathbb{N}$, we define

$$
s_{i, j}:=q_{\min (i, j)}\left(q_{\max (i, j)} \alpha-p_{\max (i, j)}\right), \quad A_{i}:=\sum_{j=0}^{\infty} b_{j} s_{i, j} .
$$

Noting that $a_{i+1} s_{i, j}=s_{i+1, j}-s_{i-1, j}+(-1)^{i} \delta_{i, j}$ for $i, j \geq 0$, we get

$$
a_{i+1} A_{i}=A_{i+1}-A_{i-1}+(-1)^{i} b_{i} \quad \text { for all } i \geq 0 .
$$

Lemma 1. For $1 \leq i \leq m$ we have:

(i) $\sum_{j=0}^{i-1} b_{j} q_{j}<q_{i}$.

(ii) $\left|\sum_{j=i}^{m} b_{j}\left(q_{j} \alpha-p_{j}\right)\right| \leq 1 / q_{i}$, and if $b_{i} \neq 0$, then

$$
\operatorname{sgn}\left(\sum_{j=i}^{m} b_{j}\left(q_{j} \alpha-p_{j}\right)\right)=(-1)^{i} \text {. }
$$

(iii) $\left|A_{i}\right|<1$.

(iv) If $2 \mid i$ and $b_{i}>0$, then $A_{i}>0$.

(v) If $2 \nmid i$ and $b_{i}>0$, then $A_{i}<0$.

Proof. (i) We omit the simple proof.

(ii) and (iii). For a proof see [4, Section 3, Proposition 1], and note that the $A_{i}$ there has to be replaced by $A_{i} / q_{i}$.

(iv) We have

$$
A_{i}=\sum_{j=0}^{i-1} b_{j} q_{j}\left(q_{i} \alpha-p_{i}\right)+q_{i} \sum_{j=i}^{m} b_{j}\left(q_{j} \alpha-p_{j}\right) .
$$

The first sum is non-negative since $q_{i} \alpha-p_{i} \geq 0$ if $2 \mid i$, and using (ii) we find that the second sum is positive, giving $A_{i}>0$.

(v) Similarly to (iv) noting that $q_{i} \alpha-p_{i} \leq 0$ if $2 \nmid i$.

Lemma 2. For $i \in \mathbb{N}$ we have:

(i) If $A_{i} \geq 0$ then $A_{i}=\left\{N q_{i} \alpha\right\}$.

(ii) If $A_{i}<0$ then $1+A_{i}=\left\{N q_{i} \alpha\right\}$. 
Proof. We have

$$
\begin{aligned}
A_{i}-N q_{i} \alpha & =\sum_{j=0}^{i} b_{j} q_{j}\left(q_{i} \alpha-p_{i}\right)+\sum_{j=i+1}^{m} b_{j} q_{i}\left(q_{j} \alpha-p_{j}\right)-\sum_{j=0}^{m} b_{j} q_{j} q_{i} \alpha \\
& =-\sum_{j=0}^{i} b_{j} q_{j} p_{i}-\sum_{j=i+1}^{m} b_{j} q_{i} p_{j} \in \mathbb{Z} .
\end{aligned}
$$

Hence, as $\left|A_{i}\right|<1$, we get $A_{i}=\left\{N q_{i} \alpha\right\}$ if $A_{i} \geq 0$, and $A_{i}+1=\left\{N q_{i} \alpha\right\}$ if $A_{i}<0$.

Proposition 1. (i) If $2 \mid i$ and $b_{i}>0$ then $b_{i}=a_{i+1}\left\{N q_{i} \alpha\right\}+O(1)$.

(ii) If $2 \nmid i$ and $b_{i}>0$ then $b_{i}=a_{i+1}\left(1-\left\{N q_{i} \alpha\right\}\right)+O(1)$.

In both cases the $O$-constants do not depend on $\alpha$.

Proof. Define $\alpha_{i}(\alpha)=\left[a_{i}(\alpha) ; a_{i+1}(\alpha), a_{i+2}(\alpha), \ldots\right]$. It follows that $\alpha_{i}=$ $a_{i}+1 / \alpha_{i+1}$.

(i) For $2 \mid i$, we have

$$
\begin{gathered}
\sum_{j=0}^{i-1} s_{i, j} b_{j}=\sum_{j=0}^{i-1}\left(q_{i} \alpha-p_{i}\right) q_{j} b_{j} \leq\left(q_{i} \alpha-p_{i}\right) q_{i} \leq \frac{q_{i}}{q_{i+1}} \leq \frac{1}{a_{i+1}} \\
\left|\sum_{j=i+1}^{\infty} s_{i, j} b_{j}\right| \leq \frac{q_{i}}{q_{i+1}}
\end{gathered}
$$

hence $\sum_{j=i+1}^{\infty} s_{i, j} b_{j}=O\left(1 / a_{i+1}\right)$ and furthermore

$s_{i, i}=q_{i}\left(q_{i} \alpha-p_{i}\right)=\frac{(-1)^{i} q_{i}}{q_{i} \alpha_{i+1}+q_{i-1}}=\frac{q_{i}}{q_{i} a_{i+1}+q_{i} / \alpha_{i+2}+q_{i-1}}=\frac{1}{a_{i+1}}+O(1)$.

So, $A_{i}=b_{i} / a_{i+1}+O\left(1 / a_{i+1}\right)+O\left(1 / a_{i+1}\right)$ and hence by Lemmas 1 and 2 ,

$$
b_{i}=a_{i+1}\left\{N q_{i} \alpha\right\}+O(1) .
$$

(ii) Similarly, $-A_{i}=b_{i} / a_{i+1}+O\left(1 / a_{i+1}\right)$, and again from Lemmas 1 and 2 ,

$$
b_{i}=a_{i+1}\left(1-\left\{N q_{i} \alpha\right\}\right)+O(1)
$$

By Proposition 1 we have explicit formulas for $b_{i}$ except in the case $2 \mid i$ and $A_{i}<0$ and the case $2 \nmid i$ and $A_{i}>0$. Therefore we define the exceptional sets

$$
A_{N, i}= \begin{cases}\left\{\alpha \in \Omega: A_{i}<0\right\} & \text { for } 2 \mid i, \\ \left\{\alpha \in \Omega: A_{i}>0\right\} & \text { for } 2 \nmid i .\end{cases}
$$

Note that if $2 \mid i$ and $A_{i}<0$, then $1+A_{i}=\left\{N q_{i} \alpha\right\}$, hence $0<1-\left\{N q_{i} \alpha\right\}=$ $-A_{i}<1 / a_{i+1}$. Therefore, $A_{N, i} \subseteq\left\{\alpha \in \Omega: 1-\left\{N q_{i} \alpha\right\}<1 / a_{i+1}\right\}$ for $2 \mid i$. Analogously, $A_{N, i} \subseteq\left\{\alpha \in \Omega:\left\{N q_{i} \alpha\right\}<1 / a_{i+1}\right\}$ if $2 \nmid i$. For $x \in \mathbb{R}$, consider $B(x)=\left(\{x\}-\{x\}^{2}\right) / 2$. Then we have 
LEMMA 3. If $a$ and $i \neq 0$ are real numbers, we have:

(i) $\int_{0}^{a}\{i \alpha\} d \alpha=a / 2-i^{-1} B(i a)$.
(ii) $\int_{0}^{a}(1-\{i \alpha\}) d \alpha=a / 2+i^{-1} B(-i a)$.

We omit the simple proof.

Lemma 4. (i) For even i, we have

$$
\begin{aligned}
\int_{J(\mathbf{a})}\left\{N q_{i}(\alpha) \alpha\right\} d \alpha= & \frac{1}{2 Q_{i+1}(\mathbf{a})\left(Q_{i+1}(\mathbf{a})+Q_{i}(\mathbf{a})\right)} \\
& -\frac{1}{N Q_{i}(\mathbf{a})}\left(B\left(\frac{N}{Q_{i+1}(\mathbf{a})}\right)-B\left(\frac{N}{Q_{i+1}(\mathbf{a})+Q_{i}(\mathbf{a})}\right)\right) .
\end{aligned}
$$

(ii) For odd i, we have

$$
\begin{aligned}
\int_{J(\mathbf{a})}\left(1-\left\{N q_{i}(\alpha) \alpha\right\}\right) d \alpha & =\frac{1}{2 Q_{i+1}(\mathbf{a})\left(Q_{i+1}(\mathbf{a})+Q_{i}(\mathbf{a})\right)} \\
& -\frac{1}{N Q_{i}(\mathbf{a})}\left(B\left(\frac{N}{Q_{i+1}(\mathbf{a})}\right)-B\left(\frac{N}{Q_{i+1}(\mathbf{a})+Q_{i}(\mathbf{a})}\right)\right) .
\end{aligned}
$$

Proof. We note that

$$
N Q_{i}(\mathbf{a}) \frac{P_{i+1}(\mathbf{a})}{Q_{i+1}(\mathbf{a})}=N \frac{(-1)^{i}+P_{i}(\mathbf{a}) Q_{i+1}(\mathbf{a})}{Q_{i+1}(\mathbf{a})} \equiv(-1)^{i} \frac{N}{Q_{i+1}(\mathbf{a})}(\bmod 1)
$$

and

$$
\begin{aligned}
N Q_{i}(\mathbf{a}) \frac{P_{i+1}(\mathbf{a})+P_{i}(\mathbf{a})}{Q_{i+1}(\mathbf{a})+Q_{i}(\mathbf{a})} & =N \frac{(-1)^{i}+P_{i}(\mathbf{a}) Q_{i+1}(\mathbf{a})+P_{i}(\mathbf{a}) Q_{i}(\mathbf{a})}{Q_{i+1}(\mathbf{a})+Q_{i}(\mathbf{a})} \\
& \equiv(-1)^{i} \frac{N}{Q_{i+1}(\mathbf{a})+Q_{i}(\mathbf{a})}(\bmod 1) .
\end{aligned}
$$

Consider the case of even $i$. From Lemma 3 we get

$\int\left\{N q_{i}(\alpha) \alpha\right\} d \alpha$

$J(\mathbf{a})$

$$
\begin{aligned}
= & \int_{0}^{\frac{P_{i+1}(\mathbf{a})}{Q_{i+1}(\mathbf{a})}}\left\{N q_{i}(\alpha) \alpha\right\} d \alpha-\int_{0}^{\frac{P_{i+1}(\mathbf{a})+P_{i}(\mathbf{a})}{Q_{i+1}(\mathbf{a})+Q_{i}(\mathbf{a})}}\left\{N q_{i}(\alpha) \alpha\right\} d \alpha \\
= & \frac{P_{i+1}(\mathbf{a})}{2 Q_{i+1}(\mathbf{a})}-\frac{1}{N Q_{i}(\mathbf{a})} B\left(N Q_{i}(\mathbf{a}) \frac{P_{i+1}(\mathbf{a})}{Q_{i+1}(\mathbf{a})}\right) \\
& -\left[\frac{P_{i+1}(\mathbf{a})+P_{i}(\mathbf{a})}{2\left(Q_{i+1}(\mathbf{a})+Q_{i}(\mathbf{a})\right)}-\frac{1}{N Q_{i}(\mathbf{a})} B\left(N Q_{i}(\mathbf{a}) \frac{P_{i+1}(\mathbf{a})+P_{i}(\mathbf{a})}{Q_{i+1}(\mathbf{a})+Q_{i}(\mathbf{a})}\right)\right] .
\end{aligned}
$$


So, from formulas (4) and (5),

$$
\begin{aligned}
& B\left(N Q_{i}(\mathbf{a}) \frac{P_{i+1}(\mathbf{a})}{Q_{i+1}(\mathbf{a})}\right)=B\left(\frac{N}{Q_{i+1}(\mathbf{a})}\right) \\
& B\left(N Q_{i}(\mathbf{a}) \frac{P_{i+1}(\mathbf{a})+P_{i}(\mathbf{a})}{Q_{i+1}(\mathbf{a})+Q_{i}(\mathbf{a})}\right)=B\left(\frac{N}{Q_{i+1}(\mathbf{a})+Q_{i}(\mathbf{a})}\right) .
\end{aligned}
$$

Thus

$$
\begin{aligned}
\int_{J(\mathbf{a})}\left\{N q_{i}(\alpha) \alpha\right\} d \alpha= & \frac{1}{2 Q_{i+1}(\mathbf{a})\left(Q_{i+1}(\mathbf{a})+Q_{i}(\mathbf{a})\right)} \\
& -\frac{1}{N Q_{i}(\mathbf{a})}\left(B\left(\frac{N}{Q_{i+1}(\mathbf{a})}\right)-B\left(\frac{N}{Q_{i+1}(\mathbf{a})+Q_{i}(\mathbf{a})}\right)\right) .
\end{aligned}
$$

This proves (i). The proof of (ii) is completely similar.

Proposition 2. (i) For even $i$ we have

$$
\int_{0}^{1} a_{i+1}(\alpha)\left\{N q_{i}(\alpha) \alpha\right\} d \alpha=\frac{1}{2} \int_{0}^{1}\left(1+\frac{q_{i-1}(\alpha)}{q_{i}(\alpha)}\right) \log ^{+}\left(\frac{N}{q_{i}(\alpha)}\right) d \alpha+O(1) .
$$

(ii) For odd $i$ we have

$$
\begin{aligned}
\int_{0}^{1} a_{i+1}(\alpha)\left(1-\left\{N q_{i}(\alpha) \alpha\right\}\right) d \alpha & \\
= & \frac{1}{2} \int_{0}^{1}\left(1+\frac{q_{i-1}(\alpha)}{q_{i}(\alpha)}\right) \log ^{+}\left(\frac{N}{q_{i}(\alpha)}\right) d \alpha+O(1) .
\end{aligned}
$$

Proof. For $\alpha \in \mathbb{N}^{i}$ and $a \in \mathbb{N}$ let $J(\mathbf{a}, a):=\left\{\alpha \in J(\mathbf{a}): a_{i+1}(\alpha)=a\right\}$. Then $J(\mathbf{a})=\bigcup_{a=1}^{\infty} J(\mathbf{a}, a)$ and therefore

$$
\int_{J(\mathbf{a})} a_{i+1}(\alpha)\left\{N q_{i}(\alpha) \alpha\right\} d \alpha=\sum_{a=1}^{\infty} a \int_{J(\mathbf{a}, a)}\left\{N q_{i}(\alpha) \alpha\right\} d \alpha
$$

since, when $\alpha$ runs through $J(\mathbf{a}, a), a_{i+1}(\alpha)=a$ does not depend on $\alpha$. Analogously,

$$
\int_{J(\mathbf{a})} a_{i+1}\left(1-\left\{N q_{i}(\alpha) \alpha\right\}\right) d \alpha=\sum_{a=1}^{\infty} a \int_{J(\mathbf{a}, a)}\left(1-\left\{N q_{i}(\alpha) \alpha\right\}\right) d \alpha .
$$

Furthermore, if we put $\mathbf{a}^{\prime}=(\mathbf{a}, a)$ then $Q_{k}\left(\mathbf{a}^{\prime}\right)=Q_{k}(\mathbf{a})$ for $0 \leq k \leq i$ and $Q_{i+1}\left(\mathbf{a}^{\prime}\right)=a Q_{i}(\mathbf{a})+Q_{i-1}(\mathbf{a})$. In order to calculate the sum in (8), we use Lemma 4 and the Abel summation formula. Note also that if $N \geq Q_{i}(\mathbf{a})$ 
and $T=\left[\frac{N-Q_{i-1}(\mathbf{a})}{Q_{i}(\mathbf{a})}\right]$, then

$$
\begin{aligned}
& \sum_{a=T+1}^{\infty} \frac{1}{\left(a Q_{i}(\mathbf{a})+Q_{i-1}(\mathbf{a})\right)^{2}} \\
& \quad=\left[(T+1) Q_{i}(\mathbf{a})+Q_{i-1}(\mathbf{a})\right]^{-2}+\sum_{a=T+2}^{\infty} \int_{a-1}^{a} \frac{d x}{\left(a Q_{i}(\mathbf{a})+Q_{i-1}(\mathbf{a})\right)^{2}} \\
& \quad \leq\left[\frac{N-Q_{i-1}(\mathbf{a})}{Q_{i}(\mathbf{a})} \cdot Q_{i}(\mathbf{a})+Q_{i-1}(\mathbf{a})\right]^{-2}+\sum_{a=T+2}^{\infty} \int_{a-1}^{a} \frac{d x}{\left(a Q_{i}(\mathbf{a})+Q_{i-1}(\mathbf{a})\right)^{2}} \\
& \quad \leq N^{-2}+\sum_{a=T+2}^{\infty} \int_{a-1}^{a} \frac{-1}{\left(x Q_{i}(\mathbf{a})+Q_{i-1}(\mathbf{a})\right)^{2}} \\
& \quad \leq N^{-1} Q_{i}^{-1}(\mathbf{a})+\left[\frac{1}{Q_{i}(\mathbf{a})\left(x Q_{i}(\mathbf{a})+Q_{i-1}(\mathbf{a})\right)}\right]_{T+1}^{\infty}=O\left(\frac{1}{N Q_{i}(\mathbf{a})}\right) .
\end{aligned}
$$

If $Q_{i-1}(\mathbf{a})<N<Q_{i}(\mathbf{a})$, we have $T=0$, so

$$
\begin{aligned}
& \sum_{a=1}^{\infty} \frac{1}{\left(a Q_{i}(\mathbf{a})+Q_{i-1}(\mathbf{a})\right)^{2}} \\
& \quad=\left[Q_{i}(\mathbf{a})+Q_{i-1}(\mathbf{a})\right]^{-2}+\sum_{a=2}^{\infty} \int_{a-1}^{a} \frac{d x}{\left(a Q_{i}(\mathbf{a})+Q_{i-1}(\mathbf{a})\right)^{2}} \\
& \quad \leq Q_{i}(\mathbf{a})^{-2}+\sum_{a=2}^{\infty} \int_{a-1}^{a} \frac{d x}{\left(x Q_{i}(\mathbf{a})+Q_{i-1}(\mathbf{a})\right)^{2}} \\
& \quad \leq N^{-1} Q_{i}(\mathbf{a})^{-1}+\left[\frac{-1}{Q_{i}(\mathbf{a})\left(x Q_{i}(\mathbf{a})+Q_{i-1}(\mathbf{a})\right)}\right]_{1}^{\infty}=O\left(\frac{1}{N Q_{i}(\mathbf{a})}\right)
\end{aligned}
$$

Next we estimate the sum (8) from $a=T+1$ to $\infty$ from above. As $a \geq T+1$ we get

$$
a>\frac{N-Q_{i-1}(\mathbf{a})}{Q_{i}(\mathbf{a})} \quad \text { and hence } \quad 0 \leq \frac{N}{a Q_{i}(\mathbf{a})+Q_{i-1}(\mathbf{a})}<1
$$

which implies that $N / Q_{i+1}(\mathbf{a})$ and $N /\left(Q_{i+1}(\mathbf{a})+Q_{i}(\mathbf{a})\right)$ lie in the interval $(0,1)$. Note also that for $x \in(0,1), B(x)=\left(x-x^{2}\right) / 2$. It follows that

$$
\begin{aligned}
& \sum_{a=T+1}^{\infty} a \int_{J(\mathbf{a}, a)}\left\{N q_{i}(\alpha) \alpha\right\} d \alpha \\
& \quad=\sum_{a=T+1}^{\infty} a\left(\frac{1}{2\left(a Q_{i}(\mathbf{a})+Q_{i-1}(\mathbf{a})\right)\left((a+1) Q_{i}(\mathbf{a})+Q_{i-1}(\mathbf{a})\right)}\right)
\end{aligned}
$$




$$
\begin{aligned}
-\sum_{a=T+1}^{\infty} a\left(\frac { 1 } { N Q _ { i } ( \mathbf { a } ) } \left(B\left(\frac{N}{a Q_{i}(\mathbf{a})+Q_{i-1}(\mathbf{a})}\right)\right.\right. \\
\left.\left.\quad-B\left(\frac{N}{(a+1) Q_{i}(\mathbf{a})+Q_{i-1}(\mathbf{a})}\right)\right)\right) \\
=\sum_{a=T+1}^{\infty} \frac{a N}{2 Q_{i}(\mathbf{a})}\left(\frac{1}{\left(a Q_{i}(\mathbf{a})+Q_{i-1}(\mathbf{a})\right)^{2}}-\frac{1}{\left((a+1) Q_{i}(\mathbf{a})+Q_{i-1}(\mathbf{a})\right)^{2}}\right) \\
=\frac{1}{2 Q_{i}(\mathbf{a})}\left(\sum_{a=T+1}^{\infty} \frac{T}{\left(a Q_{i}(\mathbf{a})+Q_{i-1}(\mathbf{a})\right)^{2}}+\frac{N / Q_{i}(\mathbf{a})}{\left((T+1) Q_{i}(\mathbf{a})+Q_{i-1}(\mathbf{a})\right)^{2}}\right) \\
=O\left(\frac{N}{N_{i}(\mathbf{a})}\left(\frac{1}{N Q_{i}(\mathbf{a})}+\frac{1}{Q_{i}(\mathbf{a})^{2}}\right)=O(\lambda(J(\mathbf{a}))) .\right.
\end{aligned}
$$

Furthermore

$$
\begin{aligned}
& \sum_{a=1}^{T} \frac{a}{N Q_{i}(\mathbf{a})}\left(B\left(\frac{N}{a Q_{i}(\mathbf{a})+Q_{i-1}(\mathbf{a})}\right)-B\left(\frac{N}{(a+1) Q_{i}(\mathbf{a})+Q_{i-1}(\mathbf{a})}\right)\right) \\
& =\frac{1}{N Q_{i}(\mathbf{a})}\left(\sum_{a=1}^{T} B\left(\frac{N}{a Q_{i}(\mathbf{a})+Q_{i-1}(\mathbf{a})}\right)-T B\left(\frac{N}{(T+1) Q_{i}(\mathbf{a})+Q_{i-1}(\mathbf{a})}\right)\right) \\
& =O\left(\frac{T}{N Q_{i}(\mathbf{a})}\right)=O\left(\frac{1}{Q_{i}(\mathbf{a})^{2}}\right)=O(\lambda(J(\mathbf{a}))) .
\end{aligned}
$$

Therefore

$$
\begin{aligned}
& \int_{J(\mathbf{a})} a_{i+1}(\alpha)\left\{N q_{i}(\alpha) \alpha\right\} d \alpha \\
& =\frac{1}{2} \sum_{a=1}^{T} \frac{a}{Q_{i}(\mathbf{a})}\left(\frac{1}{a Q_{i}(\mathbf{a})+Q_{i-1}(\mathbf{a})}-\frac{1}{(a+1) Q_{i}(\mathbf{a})+Q_{i-1}(\mathbf{a})}\right) \\
& \quad-\sum_{a=1}^{T} \frac{a}{N Q_{i}(\mathbf{a})}\left(B\left(\frac{N}{a Q_{i}(\mathbf{a})+Q_{i-1}(\mathbf{a})}\right)-B\left(\frac{N}{(a+1) Q_{i}(\mathbf{a})+Q_{i-1}(\mathbf{a})}\right)\right) \\
& \quad+\sum_{a=T+1}^{\infty} \int_{J(\mathbf{a}, a)}\left\{N q_{i}(\alpha) \alpha\right\} d \alpha \\
& =\frac{1}{2 Q_{i}(\mathbf{a})} \sum_{a=1}^{T} \frac{1}{a Q_{i}(\mathbf{a})+Q_{i-1}(\mathbf{a})}-\frac{T}{2 Q_{i}(\mathbf{a})} \frac{1}{(T+1) Q_{i}(\mathbf{a})+Q_{i-1}(\mathbf{a})} \\
& \quad+O(\lambda(J(\mathbf{a})))
\end{aligned}
$$




$$
\begin{aligned}
= & \frac{1}{2 Q_{i}(\mathbf{a})}\left(\sum_{a=1}^{T} \frac{1}{a Q_{i}(\mathbf{a})}-\sum_{a=1}^{T} \frac{Q_{i-1}(\mathbf{a})}{a Q_{i}(\mathbf{a})\left(a Q_{i}(\mathbf{a})+Q_{i-1}(\mathbf{a})\right)}\right) \\
& -\frac{T}{2 Q_{i}(\mathbf{a})} \frac{1}{(T+1) Q_{i}(\mathbf{a})+Q_{i-1}(\mathbf{a})}+O(\lambda(J(\mathbf{a}))) \\
= & \frac{1}{2 Q_{i}(\mathbf{a})} \sum_{a=1}^{T} \frac{1}{a Q_{i}(\mathbf{a})} \\
& +O\left(\frac{1}{Q_{i}(\mathbf{a})} \sum_{a=1}^{T} \frac{Q_{i-1}(\mathbf{a})}{a Q_{i}(\mathbf{a})\left(a Q_{i}(\mathbf{a})+Q_{i-1}(\mathbf{a})\right)}+\lambda(J(\mathbf{a}))\right) \\
= & \frac{1}{2 Q_{i}(\mathbf{a})^{2}} \sum_{a=1}^{T} \frac{1}{a}+O\left(\frac{1}{Q_{i}(\mathbf{a})^{2}}+\lambda(J(\mathbf{a}))\right) .
\end{aligned}
$$

Observe that

$$
\sum_{a \leq T} \frac{1}{a}=\sum_{a Q_{i}(\mathbf{a}) \leq N} \frac{1}{a}-\sum_{N-Q_{i-1}(\mathbf{a})<a Q_{i}(\mathbf{a}) \leq N} \frac{1}{a}=\sum_{a Q_{i}(\mathbf{a}) \leq N} \frac{1}{a}+O(1),
$$

as the condition $N-Q_{i-1}(\mathbf{a})<a \leq N$ is satisfied for at most one $a$. Then

$$
\begin{aligned}
& \int_{J(\mathbf{a})} a_{i+1}(\alpha)\left\{N q_{i}(\alpha) \alpha\right\} d \alpha \\
= & \frac{1}{2 Q_{i}(\mathbf{a})^{2}} \sum_{a=1}^{T} \frac{1}{a}+O\left(\frac{1}{Q_{i}(\mathbf{a})^{2}}+\lambda(J(\mathbf{a}))\right) \\
= & \frac{1}{2 Q_{i}(\mathbf{a})^{2}} \sum_{a Q_{i}(\mathbf{a}) \leq N} \frac{1}{a}+O(\lambda(J(\mathbf{a}))) \\
= & \frac{1}{2 Q_{i}(\mathbf{a})\left(Q_{i}(\mathbf{a})+Q_{i-1}(\mathbf{a})\right)}\left(1+\frac{Q_{i-1}(\mathbf{a})}{Q_{i}(\mathbf{a})} \sum_{a Q_{i}(\mathbf{a}) \leq N} \frac{1}{a}+O(\lambda(J(\mathbf{a})))\right. \\
= & \frac{1}{2} \int_{J(\mathbf{a})}\left(1+\frac{q_{i-1}(\alpha)}{q_{i}(\alpha)}\right) \sum_{a Q_{i}(\mathbf{a}) \leq N} \frac{1}{a} d \alpha+O(\lambda(J(\mathbf{a}))) \\
= & \frac{1}{2} \int_{J(\mathbf{a})}\left(1+\frac{q_{i-1}(\alpha)}{q_{i}(\alpha)}\right)\left(\log ^{+}\left(\frac{N}{q_{i}(\alpha)}\right)+O(1)\right) d \alpha+O(\lambda(J(\mathbf{a}))) \\
= & \frac{1}{2} \int_{J(\mathbf{a})}\left(1+\frac{q_{i-1}(\alpha)}{q_{i}(\alpha)}\right) \log ^{+}\left(\frac{N}{q_{i}(\alpha)}\right) d \alpha+O(\lambda(J(\mathbf{a}))) .
\end{aligned}
$$


If $N \leq Q_{i-1}(\mathbf{a})$ we have

Hence

$$
\frac{N}{Q_{i+1}(\mathbf{a})} \leq 1, \quad \frac{N}{Q_{i+1}(\mathbf{a})+Q_{i}(\mathbf{a})} \leq 1
$$

$$
\begin{aligned}
\sum_{a=1}^{\infty} a \int_{J(\mathbf{a}, a)}\left\{N q_{i}(\alpha) \alpha\right\} d \alpha & =\frac{N}{2 Q_{i}(\mathbf{a})} \sum_{a=1}^{\infty} \frac{1}{\left(a Q_{i}(\mathbf{a})+Q_{i-1}(\mathbf{a})\right)^{2}} \\
& \leq \frac{N}{2 Q_{i}(\mathbf{a})} \sum_{a=1}^{\infty} \frac{1}{a^{2} N Q_{i}(\mathbf{a})}=\frac{1}{2 Q_{i}(\mathbf{a})^{2}} \sum_{a=1}^{\infty} \frac{1}{a^{2}} \\
& =O(\lambda(J(\mathbf{a})))
\end{aligned}
$$

As $N \leq Q_{i-1}(\mathbf{a})$ we have $\log ^{+}\left(N / q_{i}(\alpha)\right)=0$ and formula (9) is valid in this case also. By summing up in (9) over all $\mathbf{a} \in \mathbb{N}^{i}$ we get (6). Analogously we obtain (7) for odd $i$.

Proposition 3. There exists a constant $C>0$ such that for all $N$ and $i$,

$$
\int_{A_{N, i}} a_{i+1}(\alpha) d \alpha \leq C
$$

Proof. First we treat the case of $i$ odd. We have

$$
\begin{aligned}
\int_{A_{N, i}} a_{i+1}(\alpha) d \alpha & =\sum_{\mathbf{a} \in \mathbb{N}^{i}} \sum_{a=1}^{\infty} \int_{A_{N, i} \cap J(\mathbf{a}, a)} a_{i+1}(\alpha) d \alpha \\
& =\sum_{\mathbf{a} \in \mathbb{N}^{i}} \sum_{a=1}^{\infty} \int_{A_{N, i} \cap J(\mathbf{a}, a)} a d \alpha=\sum_{\mathbf{a} \in \mathbb{N}^{i}} \sum_{a=1}^{\infty} a \lambda\left(A_{N, i} \cap J(\mathbf{a}, a)\right) .
\end{aligned}
$$

Now, for odd $i$,

$$
A_{N, i} \cap J(\mathbf{a}, a) \subseteq\left\{\alpha \in[0,1]:\left\{N q_{i}(\alpha) \alpha\right\}<1 / a\right\} \cap J(\mathbf{a}, a) .
$$

Consider the set

$$
M_{k}(\mathbf{a})=\left\{\alpha \in J(\mathbf{a}): k=\left[N q_{i}(\alpha) \alpha\right]\right\}
$$

Then

$$
A_{N, i} \cap J(\mathbf{a}, a)=\bigcup_{k=0}^{N Q_{i}(\mathbf{a})-1}\left(A_{N, i} \cap J(\mathbf{a}, a) \cap M_{k}(\mathbf{a})\right)
$$

and hence

$$
\int_{A_{N, i}} a_{i+1}(\alpha) d \alpha=\sum_{\mathbf{a} \in \mathbb{N}^{i}} \sum_{a=1}^{\infty} a \sum_{0 \leq k \leq N Q_{i}(\mathbf{a})} \lambda\left(A_{N, i} \cap J(\mathbf{a}, a) \cap M_{k}(\mathbf{a})\right) .
$$

If $\alpha \in A_{N, i} \cap J(\mathbf{a}, a) \cap M_{k}(\mathbf{a})$ then

$$
\frac{k}{N Q_{i}(\mathbf{a})}<\alpha<\frac{k}{N Q_{i}(\mathbf{a})}+\frac{1}{a N Q_{i}(\mathbf{a})}
$$


and

$$
\frac{a P_{i}(\mathbf{a})+P_{i-1}(\mathbf{a})}{a Q_{i}(\mathbf{a})+Q_{i-1}(\mathbf{a})} \leq \alpha \leq \frac{(a+1) P_{i}(\mathbf{a})+P_{i-1}(\mathbf{a})}{(a+1) Q_{i}(\mathbf{a})+Q_{i-1}(\mathbf{a})} .
$$

We now define, omitting the dependence on a in $P_{i}$ and $Q_{i}$,

$$
\begin{aligned}
& E_{1}(\mathbf{a}, a)=\left\{k \in \mathbb{Z}_{+}: \frac{k}{N Q_{i}} \leq \frac{a P_{i}+P_{i-1}}{a Q_{i}+Q_{i-1}} \leq \frac{(a+1) P_{i}+P_{i-1}}{(a+1) Q_{i}+Q_{i-1}} \leq \frac{k}{N Q_{i}}+\frac{1}{a N Q_{i}}\right\}, \\
& E_{2}(\mathbf{a}, a)=\left\{k \in \mathbb{Z}_{+}: \frac{a P_{i}+P_{i-1}}{a Q_{i}+Q_{i-1}} \leq \frac{k}{N Q_{i}} \leq \frac{k}{N Q_{i}}+\frac{1}{a N Q_{i}} \leq \frac{(a+1) P_{i}+P_{i-1}}{(a+1) Q_{i}+Q_{i-1}}\right\}, \\
& E_{3}(\mathbf{a}, a)=\left\{k \in \mathbb{Z}_{+}: \frac{k}{N Q_{i}} \leq \frac{a P_{i}+P_{i-1}}{a Q_{i}+Q_{i-1}} \leq \frac{k}{N Q_{i}}+\frac{1}{a N Q_{i}} \leq \frac{(a+1) P_{i}+P_{i-1}}{(a+1) Q_{i}+Q_{i-1}}\right\}, \\
& E_{4}(\mathbf{a}, a)=\left\{k \in \mathbb{Z}_{+}: \frac{a P_{i}+P_{i-1}}{a Q_{i}+Q_{i-1}} \leq \frac{k}{N Q_{i}} \leq \frac{(a+1) P_{i}+P_{i-1}}{(a+1) Q_{i}+Q_{i-1}} \leq \frac{k}{N Q_{i}}+\frac{1}{a N Q_{i}}\right\} .
\end{aligned}
$$

Then

$$
\int_{A_{N, i}} a_{i+1}(\alpha) d \alpha \leq \sum_{\mathbf{a} \in \mathbb{N}^{i}} \sum_{a=1}^{\infty} a \sum_{j=1}^{4} \sum_{k \in E_{j}(\mathbf{a}, a)} \lambda\left(A_{N, i} \cap J(\mathbf{a}, a) \cap M_{k}(\mathbf{a})\right) .
$$

We first derive an upper bound for

$$
\sum_{a=1}^{\infty} a \sum_{k \in E_{1}(\mathbf{a}, a)} \lambda\left(A_{N, i} \cap J(\mathbf{a}, a) \cap M_{k}(\mathbf{a})\right) .
$$

From the conditions for $k$ in $E_{1}(\mathbf{a}, a)$ we obtain

$$
k \leq N Q_{i}(\mathbf{a}) \frac{a P_{i}(\mathbf{a})+P_{i-1}(\mathbf{a})}{a Q_{i}(\mathbf{a})+Q_{i-1}(\mathbf{a})}, \quad k \geq N Q_{i}(\mathbf{a}) \frac{(a+1) P_{i}(\mathbf{a})+P_{i-1}(\mathbf{a})}{(a+1) Q_{i}(\mathbf{a})+Q_{i-1}(\mathbf{a})}-\frac{1}{a} .
$$

Thus

$$
k \in\left[N Q_{i}(\mathbf{a}) \frac{(a+1) P_{i}(\mathbf{a})+P_{i-1}(\mathbf{a})}{(a+1) Q_{i}(\mathbf{a})+Q_{i-1}(\mathbf{a})}-\frac{1}{a}, N Q_{i}(\mathbf{a}) \frac{a P_{i}(\mathbf{a})+P_{i-1}(\mathbf{a})}{a Q_{i}(\mathbf{a})+Q_{i-1}(\mathbf{a})}\right],
$$

which is an interval of length

$$
\frac{N Q_{i}(\mathbf{a})}{\left(a Q_{i}(\mathbf{a})+Q_{i-1}(\mathbf{a})\right)\left((a+1) Q_{i}(\mathbf{a})+Q_{i-1}(\mathbf{a})\right)}+\frac{1}{a} .
$$

If $E_{1}(\mathbf{a}, a)$ is not empty we have

$$
\begin{aligned}
\frac{1}{a N Q_{i}(\mathbf{a})} & =\frac{k}{N Q_{i}(\mathbf{a})}+\frac{1}{a N Q_{i}(\mathbf{a})}-\left(\frac{k}{N Q_{i}(\mathbf{a})}\right) \\
& \geq \frac{(a+1) P_{i}(\mathbf{a})+P_{i-1}(\mathbf{a})}{(a+1) Q_{i}(\mathbf{a})+Q_{i-1}(\mathbf{a})}-\frac{a P_{i}(\mathbf{a})+P_{i-1}(\mathbf{a})}{a Q_{i}(\mathbf{a})+Q_{i-1}(\mathbf{a})} \\
& =\frac{1}{\left(a Q_{i}(\mathbf{a})+Q_{i-1}(\mathbf{a})\right)\left((a+1) Q_{i}(\mathbf{a})+Q_{i-1}(\mathbf{a})\right)}
\end{aligned}
$$

and hence 


$$
\begin{aligned}
N & \leq \frac{\left(a Q_{i}(\mathbf{a})+Q_{i-1}(\mathbf{a})\right)\left((a+1) Q_{i}(\mathbf{a})+Q_{i-1}(\mathbf{a})\right)}{a Q_{i}(\mathbf{a})} \\
& \leq \frac{(a+1) Q_{i}(\mathbf{a})(a+2) Q_{i}(\mathbf{a})}{a Q_{i}(\mathbf{a})} \leq \frac{(a+a) Q_{i}(\mathbf{a})(a+2 a) Q_{i}(\mathbf{a})}{a Q_{i}(\mathbf{a})} \\
& \leq 6 a Q_{i}(\mathbf{a})
\end{aligned}
$$

We have $P_{i}(\mathbf{a}) Q_{i-1}(\mathbf{a})-P_{i-1}(\mathbf{a}) Q_{i}(\mathbf{a})=1$. It follows for $k \in E_{1}(\mathbf{a}, a)$ that

$$
\begin{aligned}
k & \leq N Q_{i}(\mathbf{a}) \frac{a P_{i}(\mathbf{a})+P_{i-1}(\mathbf{a})}{a Q_{i}(\mathbf{a})+Q_{i-1}(\mathbf{a})} \\
& =N \frac{a P_{i}(\mathbf{a}) Q_{i}(\mathbf{a})+P_{i-1}(\mathbf{a}) Q_{i}(\mathbf{a})}{a Q_{i}(\mathbf{a})+Q_{i-1}(\mathbf{a})} \\
& =N \frac{a P_{i}(\mathbf{a}) Q_{i}(\mathbf{a})-1+Q_{i-1}(\mathbf{a}) P_{i}(\mathbf{a})}{a Q_{i}(\mathbf{a})+Q_{i-1}(\mathbf{a})} \\
& =N P_{i}(\mathbf{a}) \frac{a Q_{i}(\mathbf{a})+Q_{i-1}(\mathbf{a})}{a Q_{i}(\mathbf{a})+Q_{i-1}(\mathbf{a})}-\frac{N}{a Q_{i}(\mathbf{a})+Q_{i-1}(\mathbf{a})} \\
& =N P_{i}(\mathbf{a})-\frac{N}{a Q_{i}(\mathbf{a})+Q_{i-1}(\mathbf{a})}
\end{aligned}
$$

Similarly, using

$$
k \geq N Q_{i}(\mathbf{a}) \frac{(a+1) P_{i}(\mathbf{a})+P_{i-1}(\mathbf{a})}{(a+1) Q_{i}(\mathbf{a})+Q_{i-1}(\mathbf{a})}-\frac{1}{a},
$$

we have

$$
k \geq N P_{i}(\mathbf{a})-\frac{N}{(a+1) Q_{i}(\mathbf{a})+Q_{i-1}(\mathbf{a})}-\frac{1}{a}
$$

and hence

$$
\begin{aligned}
N P_{i}(\mathbf{a})-\frac{N}{(a+1) Q_{i}(\mathbf{a})+Q_{i-1}(\mathbf{a})} & -\frac{1}{a} \leq k \\
& \leq N P_{i}(\mathbf{a})-\frac{N}{a Q_{i}(\mathbf{a})+Q_{i-1}(\mathbf{a})} .
\end{aligned}
$$

Therefore $\left|E_{1}(\mathbf{a}, a)\right| \leq 1$. Now, if

$$
\frac{2 N}{Q_{i}(\mathbf{a})}<a, \quad \frac{N}{a Q_{i}(\mathbf{a})+Q_{i-1}(\mathbf{a})}<\frac{1}{2}
$$

we directly get, for $a>1$,

$$
\frac{N}{(a+1) Q_{i}(\mathbf{a})+Q_{i-1}(\mathbf{a})}+\frac{1}{a}<\frac{1}{a}+\frac{1}{2}<1,
$$

which means that the interval (13) does not contain an integer in this case. 
Furthermore

$$
\begin{aligned}
\lambda(J(\mathbf{a}, a)) & =\frac{1}{\left(a Q_{i}(\mathbf{a})+Q_{i-1}(\mathbf{a})\right)\left((a+1) Q_{i}(\mathbf{a})+Q_{i-1}(\mathbf{a})\right)} \\
& \leq \frac{1}{a^{2}\left(Q_{i}(\mathbf{a})\right)^{2}}=\frac{2}{2 a^{2}\left(Q_{i}(\mathbf{a})\right)^{2}} \\
& \leq \frac{2}{a^{2} Q_{i}(\mathbf{a})\left(Q_{i}(\mathbf{a})+Q_{i-1}(\mathbf{a})\right)}=2 \frac{\lambda(J(\mathbf{a}))}{a^{2}} .
\end{aligned}
$$

Thus, since for positive integers $k$ and $m$,

$$
\sum_{a=k}^{m k} \frac{1}{a} \leq \sum_{a=k}^{m k} \frac{1}{k}=(m k-k+1) \frac{1}{k} \leq m,
$$

we have

$$
\begin{aligned}
& \sum_{a=1}^{\infty} a \sum_{k \in E_{1}(\mathbf{a}, a)} \lambda\left(A_{N, i} \cap J(\mathbf{a}, a) \cap M_{k}(\mathbf{a})\right) \leq \sum_{a=1}^{\infty} a \sum_{k \in E_{1}(\mathbf{a}, a)} \lambda(J(\mathbf{a}, a)) \\
& \leq 2 \lambda(J(\mathbf{a}, 1))+\sum_{N /\left(6 Q_{i}(\mathbf{a})\right) \leq a \leq 2 N / Q_{i}(\mathbf{a})} a \lambda(J(\mathbf{a}, a)) \\
& \leq \frac{2}{\left(Q_{i}(\mathbf{a})+Q_{i-1}(\mathbf{a})\right)\left(2 Q_{i}(\mathbf{a})+Q_{i-1}(\mathbf{a})\right)}+\sum_{N /\left(6 Q_{i}(\mathbf{a})\right) \leq a \leq 2 N / Q_{i}(\mathbf{a})} 2 \frac{\lambda(J(\mathbf{a}))}{a} \\
& \leq \frac{2}{\left(Q_{i}(\mathbf{a})\right)^{2}}+2 \cdot 12 \cdot \lambda(J(\mathbf{a}))=O(\lambda(J(\mathbf{a}))) .
\end{aligned}
$$

We have established

$$
\sum_{a=1}^{\infty} a \sum_{k \in E_{1}(\mathbf{a}, a)} \lambda\left(A_{N, i} \cap J(\mathbf{a}, a) \cap M_{k}(\mathbf{a})\right)=O(\lambda(J(\mathbf{a}))) .
$$

In order to estimate

$$
\sum_{a=1}^{\infty} a \sum_{k \in E_{2}(\mathbf{a}, a)} \lambda\left(A_{N, i} \cap J(\mathbf{a}, a) \cap M_{k}(\mathbf{a})\right),
$$

we start by observing that for $k$ in $E_{2}(\mathbf{a}, a)$ we have

$$
\begin{aligned}
& k \geq N Q_{i}(\mathbf{a}) \frac{a P_{i}(\mathbf{a})+P_{i-1}(\mathbf{a})}{a Q_{i}(\mathbf{a})+Q_{i-1}(\mathbf{a})}, \\
& k \leq N Q_{i}(\mathbf{a}) \frac{(a+1) P_{i}(\mathbf{a})+P_{i-1}(\mathbf{a})}{(a+1) Q_{i}(\mathbf{a})+Q_{i-1}(\mathbf{a})}-\frac{1}{a},
\end{aligned}
$$

so it follows that

$$
k \in\left[N Q_{i}(\mathbf{a}) \frac{a P_{i}(\mathbf{a})+P_{i-1}(\mathbf{a})}{a Q_{i}(\mathbf{a})+Q_{i-1}(\mathbf{a})}, N Q_{i}(\mathbf{a}) \frac{(a+1) P_{i}(\mathbf{a})+P_{i-1}(\mathbf{a})}{(a+1) Q_{i}(\mathbf{a})+Q_{i-1}(\mathbf{a})}-\frac{1}{a}\right],
$$

which is an interval of length 


$$
\frac{N Q_{i}(\mathbf{a})}{\left(a Q_{i}(\mathbf{a})+Q_{i-1}(\mathbf{a})\right)\left((a+1) Q_{i}(\mathbf{a})+Q_{i-1}(\mathbf{a})\right)}-\frac{1}{a} .
$$

As

$$
A_{N, i} \cap J(\mathbf{a}, a) \cap M_{k}(\mathbf{a}) \subseteq\left[\frac{k}{N Q_{i}(\mathbf{a})}, \frac{k}{N Q_{i}(\mathbf{a})}+\frac{1}{a N Q_{i}(\mathbf{a})}\right],
$$

we get

$$
\lambda\left(A_{N, i} \cap J(\mathbf{a}, a) \cap M_{k}(\mathbf{a})\right) \leq \frac{1}{a N Q_{i}(\mathbf{a})} .
$$

If $E_{2}(\mathbf{a}, a)$ is not empty we have

$$
\frac{1}{a N Q_{i}(\mathbf{a})} \leq \frac{1}{\left(a Q_{i}(\mathbf{a})+Q_{i-1}(\mathbf{a})\right)\left((a+1) Q_{i}(\mathbf{a})+Q_{i-1}(\mathbf{a})\right)}
$$

and hence $2 N \geq a Q_{i}(\mathbf{a})$. Moreover, from (14) we get

$$
\begin{aligned}
& N P_{i}(\mathbf{a})-\frac{N}{a Q_{i}(\mathbf{a})+Q_{i-1}(\mathbf{a})} \leq k \\
& \quad \leq N P_{i}(\mathbf{a})-\frac{N}{(a+1) Q_{i}(\mathbf{a})+Q_{i-1}(\mathbf{a})}-\frac{1}{a} .
\end{aligned}
$$

From this we infer that $\left|E_{2}(\mathbf{a}, a)\right|$ is at most

$$
\begin{aligned}
& \frac{N}{a Q_{i}(\mathbf{a})+Q_{i-1}(\mathbf{a})}-\frac{N}{(a+1) Q_{i}(\mathbf{a})+Q_{i-1}(\mathbf{a})}-\frac{1}{a}+1 \\
& =O\left(\frac{N Q_{i}(\mathbf{a})}{\left(a Q_{i}(\mathbf{a})+Q_{i-1}(\mathbf{a})\right)\left((a+1) Q_{i}(\mathbf{a})+Q_{i-1}(\mathbf{a})\right)}+1\right) \\
& =O\left(\frac{N}{a^{2} Q_{i}(\mathbf{a})}+1\right) \text {. }
\end{aligned}
$$

Consider the set

$$
A(\mathbf{a})=\left\{a \in \mathbb{N}: \frac{N Q_{i}(\mathbf{a})}{\left(a Q_{i}(\mathbf{a})+Q_{i-1}(\mathbf{a})\right)\left((a+1) Q_{i}(\mathbf{a})+Q_{i-1}(\mathbf{a})\right)} \geq \frac{1}{2}\right\} .
$$

In the case $a \in \mathbb{N} \backslash A(\mathbf{a})$, there is no $k$ satisfying (16), which means that in this case $E_{2}(\mathbf{a}, a)=\emptyset$. If $a \in A(\mathbf{a})$, we have

$$
2 N Q_{i}(\mathbf{a}) \geq\left(a Q_{i}(\mathbf{a})+Q_{i-1}(\mathbf{a})\right)\left((a+1) Q_{i}(\mathbf{a})+Q_{i-1}(\mathbf{a})\right) \geq a^{2} Q_{i}^{2}(\mathbf{a})
$$

and then $N /\left(a^{2} Q_{i}(\mathbf{a})\right) \geq 1 / 2$. This implies that $1=O\left(N /\left(a^{2} Q_{i}(\mathbf{a})\right)\right)$ and from (17) we get $\left|E_{2}(\mathbf{a}, a)\right|=O\left(N /\left(a^{2} Q_{i}(\mathbf{a})\right)\right)$. Thus

$$
\begin{aligned}
\sum_{a=1}^{\infty} a \sum_{k \in E_{2}(\mathbf{a}, a)} \lambda\left(A_{N, i} \cap J(\mathbf{a}, a) \cap M_{k}(\mathbf{a})\right) \\
=\sum_{a \leq 2 N / Q_{i}(\mathbf{a})} a \sum_{k \in E_{2}(\mathbf{a}, a)} \lambda\left(A_{N, i} \cap J(\mathbf{a}, a) \cap M_{k}(\mathbf{a})\right)
\end{aligned}
$$




$$
\begin{aligned}
& =\sum_{a \in A(\mathbf{a}) \wedge a \leq 2 N / Q_{i}(\mathbf{a})} a \sum_{k \in E_{2}(\mathbf{a}, a)} \lambda\left(A_{N, i} \cap J(\mathbf{a}, a) \cap M_{k}(\mathbf{a})\right) \\
& =O\left(\sum_{a \in A(\mathbf{a})} a \frac{N}{a^{2} Q_{i}(\mathbf{a})} \cdot \frac{1}{a N Q_{i}(\mathbf{a})}\right)=O\left(\sum_{a=1}^{\infty} \frac{1}{a^{2}\left(Q_{i}(\mathbf{a})\right)^{2}}\right) \\
& =O\left(\frac{1}{\left(Q_{i}(\mathbf{a})\right)^{2}}\right)=O(\lambda(J(\mathbf{a}))) .
\end{aligned}
$$

Hence,

$$
\sum_{a=1}^{\infty} a \sum_{k \in E_{2}(\mathbf{a}, a)} \lambda\left(A_{N, i} \cap J(\mathbf{a}, a) \cap M_{k}(\mathbf{a})\right)=O(\lambda(J(\mathbf{a}))) .
$$

In order to estimate

$$
\sum_{a=1}^{\infty} a \sum_{k \in E_{3}(\mathbf{a}, a)} \lambda\left(A_{N, i} \cap J(\mathbf{a}, a) \cap M_{k}(\mathbf{a})\right)
$$

we notice that for $k \in E_{3}(\mathbf{a}, a)$ we have

$$
k \leq N Q_{i}(\mathbf{a}) \frac{a P_{i}(\mathbf{a})+P_{i-1}(\mathbf{a})}{a Q_{i}(\mathbf{a})+Q_{i-1}(\mathbf{a})}, \quad k \geq N Q_{i}(\mathbf{a}) \frac{a P_{i}(\mathbf{a})+P_{i-1}(\mathbf{a})}{a Q_{i}(\mathbf{a})+Q_{i-1}(\mathbf{a})}-\frac{1}{a} .
$$

From these conditions we deduce that $E_{3}(\mathbf{a}, a)$ is an interval of length at most $1 / a$ and (as before) that if $k \in E_{3}(\mathbf{a}, a)$, then

$$
N P_{i}(\mathbf{a})-\frac{N}{a Q_{i}(\mathbf{a})+Q_{i-1}(\mathbf{a})}-\frac{1}{a} \leq k \leq N P_{i}(\mathbf{a})-\frac{N}{a Q_{i}(\mathbf{a})+Q_{i-1}(\mathbf{a})} .
$$

We have seen above that for $2 N / Q_{i}(\mathbf{a})<a, E_{3}(\mathbf{a}, a)$ is empty. So,

$$
\begin{aligned}
\sum_{a=1}^{\infty} a \sum_{k \in E_{3}(\mathbf{a}, a)} \lambda & \left(A_{N, i} \cap J(\mathbf{a}, a) \cap M_{k}(\mathbf{a})\right) \\
& \leq \sum_{a=1}^{\infty} a \sum_{k \in E_{3}(\mathbf{a}, a)} \lambda(J(\mathbf{a}, a)) \\
& \leq \sum_{1 \leq a \leq 2 N / Q_{i}(\mathbf{a})} a \lambda(J(\mathbf{a}, a)) \leq \sum_{1 \leq a \leq 2 N / Q_{i}(\mathbf{a})} a \frac{1}{a N Q_{i}(\mathbf{a})} \\
& \leq \frac{2 N}{Q_{i}(\mathbf{a})} \cdot \frac{1}{N Q_{i}(\mathbf{a})}=\frac{2}{Q_{i}^{2}(\mathbf{a})}=O(\lambda(J(\mathbf{a})))
\end{aligned}
$$

Hence

$$
\sum_{a=1}^{\infty} a \sum_{k \in E_{3}(\mathbf{a}, a)} \lambda\left(A_{N, i} \cap J(\mathbf{a}, a) \cap M_{k}(\mathbf{a})\right)=O(\lambda(J(\mathbf{a})))
$$


In order to estimate

$$
\sum_{a=1}^{\infty} a \sum_{k \in E_{4}(\mathbf{a}, a)} \lambda\left(A_{N, i} \cap J(\mathbf{a}, a) \cap M_{k}(\mathbf{a})\right),
$$

we observe that for $k \in E_{4}(\mathbf{a}, a)$ we have

$$
\begin{aligned}
& k \leq N Q_{i}(\mathbf{a}) \frac{(a+1) P_{i}(\mathbf{a})+P_{i-1}(\mathbf{a})}{(a+1) Q_{i}(\mathbf{a})+Q_{i-1}(\mathbf{a})} \\
& k \geq N Q_{i}(\mathbf{a}) \frac{(a+1) P_{i}(\mathbf{a})+P_{i-1}(\mathbf{a})}{(a+1) Q_{i}(\mathbf{a})+Q_{i-1}(\mathbf{a})}-\frac{1}{a} .
\end{aligned}
$$

From these conditions we deduce that $E_{4}(\mathbf{a}, a)$ is an interval of length at most $1 / a$ and, as before, if $k \in E_{4}(\mathbf{a}, a)$ then

$$
\begin{aligned}
N P_{i}(\mathbf{a})-\frac{N}{(a+1) Q_{i}(\mathbf{a})+Q_{i-1}(\mathbf{a})}-\frac{1}{a} \leq k \\
\quad \leq N P_{i}(\mathbf{a})-\frac{N}{(a+1) Q_{i}(\mathbf{a})+Q_{i-1}(\mathbf{a})} .
\end{aligned}
$$

Once again, if $2 N / Q_{i}(\mathbf{a})<a, E_{4}(\mathbf{a}, a)$ is empty. Then

$$
\begin{aligned}
\sum_{a=1}^{\infty} a \sum_{k \in E_{4}(\mathbf{a}, a)} \lambda\left(A_{N, i} \cap J(\mathbf{a}, a) \cap M_{k}(\mathbf{a})\right) & \leq \sum_{a=1}^{\infty} a \sum_{k \in E_{4}(\mathbf{a}, a)} \lambda(J(\mathbf{a}, a)) \\
& =O(\lambda(J(\mathbf{a}))) .
\end{aligned}
$$

It follows that

$$
\sum_{a=1}^{\infty} a \sum_{k \in E_{4}(\mathbf{a}, a)} \lambda\left(A_{N, i} \cap J(\mathbf{a}, a) \cap M_{k}(\mathbf{a})\right)=O(\lambda(J(\mathbf{a})) .
$$

So, if $2 \nmid i$, we have proved that

$$
\begin{aligned}
\int_{A_{N, i}} a_{i+1}(\alpha) d \alpha & =\sum_{a \in \mathbb{N}^{i}} \sum_{a=1}^{\infty} a \sum_{j=1}^{4} \sum_{k \in E_{j}(\mathbf{a}, a)} \lambda\left(A_{N, i} \cap J(\mathbf{a}, a) \cap M_{k}(\mathbf{a})\right) \\
& =\sum_{a \in \mathbb{N}^{i}} 4 \cdot O(\lambda(J(\mathbf{a})))=O(1) .
\end{aligned}
$$

The case $2 \mid i$ can be proved either similarly or by a change of variable $\alpha \rightarrow 1-\alpha$. In fact, for $0<\alpha \leq 1 / 2$ we have $a_{i+1}(1-\alpha)=a_{i}(\alpha)$ and $q_{i+1}(1-\alpha)=q_{i}(\alpha)$ for $i>0$, and if $1 / 2<\alpha \leq 1$ we have $a_{i}(1-\alpha)=a_{i+1}(\alpha)$ and $q_{i}(1-\alpha)=q_{i+1}(\alpha)$ for $i \geq 0$. Hence, 
- if $0<\alpha<1 / 2$ and $i>0$ then

$$
\begin{aligned}
1-\alpha \in A_{N, i} & \Leftrightarrow 1-\left\{N q_{i}(1-\alpha)(1-\alpha)\right\}<\frac{1}{a_{i+1}(1-\alpha)} \\
& \Leftrightarrow\left\{N q_{i}(1-\alpha) \alpha\right\}<\frac{1}{a_{i+1}(1-\alpha)} \\
& \Leftrightarrow\left\{N q_{i-1}(\alpha) \alpha\right\}<\frac{1}{a_{i}(\alpha)} \Leftrightarrow \alpha \in A_{N, i-1} ;
\end{aligned}
$$

- if $1 / 2<\alpha<1$ and $i>0$ then

$$
\begin{aligned}
1-\alpha \in A_{N, i} & \Leftrightarrow 1-\left\{N q_{i}(1-\alpha)(1-\alpha)\right\}<\frac{1}{a_{i+1}(1-\alpha)} \\
& \Leftrightarrow\left\{N q_{i}(1-\alpha) \alpha\right\}<\frac{1}{a_{i+1}(1-\alpha)} \\
& \Leftrightarrow\left\{N q_{i+1}(\alpha) \alpha\right\}<\frac{1}{a_{i+2}(\alpha)} \Leftrightarrow \alpha \in A_{N, i+1}
\end{aligned}
$$

- if $1 / 2<\alpha<1$ and $i=0$ then

$$
\begin{aligned}
1-\alpha \in A_{N, 0} & \Leftrightarrow 1-\left\{N q_{0}(1-\alpha)(1-\alpha)\right\}<\frac{1}{a_{1}(1-\alpha)} \\
& \Leftrightarrow\left\{N q_{0}(1-\alpha) \alpha\right\}<\frac{1}{a_{1}(1-\alpha)} \\
& \Leftrightarrow\left\{N q_{1}(\alpha) \alpha\right\}<\frac{1}{a_{2}(\alpha)+1} \\
& \Rightarrow\left\{N q_{1}(\alpha) \alpha\right\}<\frac{1}{a_{2}(\alpha)} \Rightarrow \alpha \in A_{N, 1} .
\end{aligned}
$$

Let $C_{X}$ be the characteristic function of the set $X$. Then for $i>0$,

$$
\begin{aligned}
\int_{A_{N, i}} a_{i+1} & (\alpha) d \alpha=\int_{0}^{1} a_{i+1}(\alpha) C_{A_{N, i}}(\alpha) d \alpha \\
& =\int_{0}^{1 / 2} a_{i+1}(1-\alpha) C_{A_{N, i}}(1-\alpha) d \alpha+\int_{1 / 2}^{1} a_{i+1}(1-\alpha) C_{A_{N, i}}(1-\alpha) d \alpha \\
& =\int_{0}^{1 / 2} a_{i}(\alpha) C_{A_{N, i-1}}(\alpha) d \alpha+\int_{1 / 2}^{1} a_{i+2}(\alpha) C_{A_{N, i+1}}(\alpha) d \alpha=O(1) .
\end{aligned}
$$

Similarly,

$$
\begin{aligned}
& \int_{A_{N, 0}} a_{1}(\alpha) d \alpha=\int_{0}^{1} a_{1}(\alpha) C_{A_{N, 0}}(\alpha) d \alpha \\
& \quad=\int_{0}^{1 / 2} a_{1}(1-\alpha) C_{A_{N, 0}}(1-\alpha) d \alpha+\int_{1 / 2}^{1} a_{1}(1-\alpha) C_{A_{N, 0}}(1-\alpha) d \alpha
\end{aligned}
$$




$$
\leq \frac{1}{2}+\int_{1 / 2}^{1}\left(a_{2}(\alpha)+1\right) C_{A_{N, 1}}(\alpha) d \alpha \leq 1+\int_{1 / 2}^{1} a_{2}(\alpha) C_{A_{N, 1}}(\alpha) d \alpha=O(1)
$$

and the result follows.

4. Proof of the Theorem. In order to prove the Theorem we start by proving the following result:

Proposition 4. Given $\alpha \in \Omega$ and $N \in \mathbb{N}$ with $N=\sum_{i=0}^{m} b_{i} q_{i}$ we have for $0 \leq i \leq m$,

$$
\int_{0}^{1} b_{i}(\alpha) d \alpha=\frac{1}{2} \int_{0}^{1}\left(1+\frac{q_{i-1}(\alpha)}{q_{i}(\alpha)}\right) \log ^{+}\left(\frac{N}{q_{i}(\alpha)}\right) d \alpha+O(1) .
$$

Proof. For even $i$ we have, by Section 2 and Propositions 1-3,

$$
\begin{aligned}
\int_{0}^{1} b_{i}(\alpha) d \alpha & =\int_{[0,1] \backslash A_{N, i}} b_{i}(N, \alpha) d \alpha+\underbrace{\int_{A_{N, i}} b_{i}(N, \alpha) d \alpha}_{=0} \\
& =\int_{[0,1] \backslash A_{N, i}}\left(a_{i+1}(\alpha)\left\{N q_{i}(\alpha) \alpha\right\}+O(1)\right) d \alpha \\
& =\int_{0}^{1} a_{i+1}(\alpha)\left\{N q_{i}(\alpha) \alpha\right\} d \alpha+O(1) \\
& =\frac{1}{2} \int_{0}^{1}\left(1+\frac{q_{i-1}(\alpha)}{q_{i}(\alpha)}\right) \log ^{+}\left(\frac{N}{q_{i}(\alpha)}\right) d \alpha+O(1) .
\end{aligned}
$$

The proof for the odd case is entirely similar.

TheOREM 1. For $N \in \mathbb{N}$,

$$
\int_{0}^{1} s_{N}(\alpha) d \alpha=\frac{1}{2} \sum_{i=0}^{\infty} \int_{0}^{1}\left(1+\frac{q_{i-1}(\alpha)}{q_{i}(\alpha)}\right) \log ^{+}\left(\frac{N}{q_{i}(\alpha)}\right) d \alpha+O(\log N) .
$$

Proof. Let $\left(F_{k}\right)_{k \geq 0}$ be the sequence of Fibonacci numbers: $F_{0}=F_{1}=1$, $F_{k+1}=F_{k}+F_{k-1}$. Then there is a $c>0$ such that $\log F_{k} \geq k / c$ for $k \geq 2$. For every $\alpha \in \Omega$ we have $q_{k}(\alpha) \geq F_{k}$, and $N \leq q_{k}(\alpha)$ for $c \log N \leq k$, and therefore for $2 \mid k$,

$$
a_{k+1}\left\{N q_{k} \alpha\right\} \leq a_{k+1} N\left|q_{k} \alpha-p_{k}\right|<a_{k+1} N / q_{k+1} \leq N / q_{k} .
$$

Similarly for $2 \nmid k$,

$$
a_{k+1}\left(1-\left\{N q_{k} \alpha\right\}\right) \leq a_{k+1} N\left|q_{k} \alpha-p_{k}\right|<N / q_{k} .
$$


Hence

$$
\sum_{2 \mid k \geq c \log N}^{\infty} a_{k+1}\left\{N q_{k} \alpha\right\}=\sum_{2 \nmid k \geq c \log N}^{\infty} a_{k+1}\left(1-\left\{N q_{k} \alpha\right\}\right)=O(1) .
$$

So, we can calculate $\int_{0}^{1} s_{N}(\alpha) d \alpha$ as follows:

$$
\begin{aligned}
& \int_{0}^{1} s_{N}(\alpha) d \alpha=\int_{0}^{1} \sum_{i=0}^{\infty} b_{i}(N, \alpha) d \alpha \\
& =\sum_{\substack{2 \mid i \\
i \leq c \log N}} \int_{[0,1] \backslash A_{N, i}} a_{i+1}(\alpha)\left\{N q_{i}(\alpha) \alpha\right\} d \alpha \\
& +\sum_{\substack{2 \nmid i \\
i \leq c \log N}} \int_{\substack{0,1] \backslash A_{N, i}\\
}} a_{i+1}(\alpha)\left(1-\left\{N q_{i}(\alpha) \alpha\right\}\right) d \alpha+O(1) \\
& =\sum_{\substack{2 \mid i \\
i \leq c \log N}}\left(\int_{0}^{1} a_{i+1}(\alpha)\left\{N q_{i}(\alpha) \alpha\right\} d \alpha+O(1)\right) \\
& +\sum_{\substack{2 \nmid i \\
i \leq c \log N}}\left(\int_{0}^{1} a_{i+1}(\alpha)\left(1-\left\{N q_{i}(\alpha) \alpha\right\}\right) d \alpha+O(1)\right)+O(1) \\
& =\sum_{\substack{2 \mid i \\
i \leq c \log N}} \int_{0}^{1} a_{i+1}(\alpha)\left\{N q_{i}(\alpha) \alpha\right\} d \alpha \\
& +\sum_{\substack{2 \nmid i \\
i \leq c \log N}} \int_{0}^{1} a_{i+1}(\alpha)\left(1-\left\{N q_{i}(\alpha) \alpha\right\}\right) d \alpha+O(\log N)+O(1) \\
& =\frac{1}{2} \sum_{i \leq c \log N} \int_{0}^{1}\left(1+\frac{q_{i-1}(\alpha)}{q_{i}(\alpha)}\right) \log ^{+}\left(\frac{N}{q_{i}(\alpha)}\right) d \alpha+O(\log N) .
\end{aligned}
$$

Since $\log ^{+}\left(N / q_{i}(\alpha)\right)=0$ for $i>c \log N$, the result follows.

Then, by Theorem 1 we have

$$
\int_{0}^{1} s_{N}(\alpha) d \alpha=\frac{1}{2} \sum_{i \leq c \log N} \int_{0}^{1}\left(1+\frac{q_{i-1}(\alpha)}{q_{i}(\alpha)}\right) \log ^{+}\left(\frac{N}{q_{i}(\alpha)}\right) d \alpha+O(\log N) .
$$

This sum has been asymptotically developed in [4] with the effect that it is equal to $\left(6 / \pi^{2}\right) \log ^{2} N+O\left((\log N)^{3 / 2} \log \log N\right)$. 
5. Concluding remarks. The methods used here can be generalized to prove that

$$
\sum_{i=0}^{\infty} \int_{0}^{1} \frac{b_{i}^{n}(N, \alpha)}{a_{i+1}^{n-1}(\alpha)} d \alpha=\frac{6}{(n+1) \pi^{2}} \log ^{2} N+O\left((\log N)^{3 / 2} \log \log N\right), \quad n \in \mathbb{N} .
$$

It seems to be hopeless to generalize this method to more general integrals, like $\int_{0}^{1} s_{N}(\alpha)^{L} d \alpha$. On the other hand it might very well happen that there is a central limit law behind our main theorem.

Acknowledgements. The result of this paper is part of my Ph.D. studies under the supervision of Johannes Schoißengeier at the Universidade de Trás-os-Montes e Alto Douro. I am very indebted to him for his valuable comments. Likewise I thank the referee for his comments and suggestions.

\section{References}

[1] M. Drmota, The distribution of patterns in digital expansions, in: Algebraic Number Theory and Diophantine Analysis (Graz, 1998), de Gruyter, 2000, 103-121.

[2] M. Drmota and J. Gajdosik, The distribution of the sum-of-digits function, J. Théor. Nombres Bordeaux 10 (1998), 17-32.

[3] M. Drmota and J. Schoißengeier, Digital expansions with respect to different bases, Monatsh. Math. 138 (2003), 31-59.

[4] J. Schoißengeier, On the discrepancy of (no), Acta Arith. 44 (1984), 241-279.

[5] - The integral mean of the discrepancy of the sequence (no), Monatsh. Math. 131 (2000), 227-234.

Departamento de Matemática

Universidade de Trás-os-Montes e Alto Douro

Quinta dos Prados, Vila Real

5001-911 Vila Real, Portugal

E-mail: rocadas@utad.pt

Received on 22.4.2002

and in revised form on 6.10.2002 